\title{
Accessible Pediatric Liver Transplantation in India: A Long way to go
}

\author{
*Mohamed Rela AND \#NARESh PShanmugham \\ From the *Institute of Liver Disease \& Transplantation, and "Institute of Advanced Pediatrics; Gleneagles Global Health City, \\ Chennai, Tamil Nadu, India. \\ *mohamed.rela@gmail.com
}

$\mathrm{O}$ ver the last decade, India has seen tremendous growth in the field of liver transplantation (LT) [1]. While pediatric LT constitute less than $10 \%$ of all transplant activity [2], extrapolating the data from the United States, its need in India is around 3000 precedures/year. However, currently less than 150 pediatric liver transplants are being performed in India annually, and the reasons for this wide gap include delayed diagnosis and referral, concerns regarding its long-term success, and the financial burden on the family. The complexity of surgical technique and peri-operative care involved in pediatric LT means that even established adult LT programs need additional skilled manpower and infrastructure to have an active pediatric LT program. While there has been a steady increase in the number of centers offering adult LT, pediatric LT remains a niche area with just around five centers performing more than 20 procedures every year.

In this issue of Indian Pediatrics, Mohan, et al. [3] report their center's experience of 200 pediatric Living Donor Liver Transplantation (pLDLT) over a 12-year period. They report a steady increase in transplant numbers over the period with results equivalent to established centers around the world. The highlight of this report is their protocol-based approach to all aspects of the peri-transplant care, including pre-operative assessment, nutritional support, post-transplant immunosuppression and post-operative care, all of which has contributed to the success of the program. This report is a testimony to the role of a multidisciplinary approach in the care of these sick children, and the authors should be congratulated for the same.

Any discussion of pediatric LT in India is incomplete without discussing the 'twin elephants in the room' - the very low deceased donation in India and the economic constraints of the average Indian family, which limits access to pediatric LT. India is a country with diverse socio-economical and family structures. While LDLT is well-established for the pediatric age group, there will always be a cohort of children who do not have suitable family donors. Mohan, et al. [3] have attempted to circumvent this problem in their program by utilizing swap donations and $\mathrm{ABO}$ incompatible transplants. However, this is not always feasible, and there will always be children left with no transplant option in such an 'LDLT or nothing' scenario. The solution to this problem lies in improving deceased donation, developing local and national organ-sharing networks, and sharing expertise in liver splitting. For example, our center established in 2010, has performed 260 pediatric LTs till date. While a majority of these are LDLT, we have performed DDLT in 31 children for whom an LDLT option was not available. Twenty-two of these children received split liver grafts, ensuring optimum utilization of each good quality liver graft for two patients.

In our experience, financial constraints remain the single most important factor that determines a child's access to this life-saving treatment in India. Young parents in the early period of earning lives, nuclear families with limited family support, lack of adequate health insurance cover and need for repeated hospital admissions, and life-long medication after transplantation are major deterrents when a family has to make a decision for the child to proceed with LT. This is compounded by the fact that a majority of centers providing this service are in the private sector with its associated high cost of treatment [4]. Unfortunately, there are no quick fix solutions to this problem. Development of pediatric LT centers in designated public hospitals is one way of dealing with this problem. This has been successful to an extent in New Delhi with the establishment of Institute of Liver and Biliary Sciences (ILBS). However, this is not universally feasible as is evident from the fact that there are very few large volume adult LT centers in the public sector. Another way of improving access is by provision of means-tested financial support for families from local authorities, charity organizations and even crowd-funding [5]. We 
have been able to transplant several children from poor economic backgrounds in the Southern states of Tamil Nadu, Andhra Pradesh and Telangana with such governmental support.

There are many who would question the ethics of promoting pediatric LT in a country where majority of children still die of easily treatable conditions such as diarrhea and malnutrition [6]. However, we believe that development of high-end treatments is an essential part of developing the overall health care infrastructure in the country. A developed health care system should provide every child and parent access to the entire gamut of facilities necessary for managing a disease. Unless these are developed by intensive government interventions, excellent single center results, such as the current report, will only cater to the tip of the iceberg of childhood liver disease in India.

Funding: None; Competing interests: None stated.

\section{REFERENCES}

1. Narasimhan G, Kota V, Rela M. Liver transplantation in India. Liver Transpl. 2016;22:1019-24.

2. US Department of Health and Human Services. Organ Procurement and Transplantation Network. Available from: https://optn.transplant.hrsa.gov/data. Accessed October 15, 2017.

3. Mohan N, Karkra S, Rastogi A, Dhaliwal MS, Raghunathan V, Goyal D, et al. Outcome of 200 pediatric living donor liver transplantations in India. Indian Pediatr. 2017;54:913-8.

4. Balarajan Y, Selvaraj S, Subramanian SV. Health care and equity in India. Lancet. 2011;377:505-15.

5. Kumar AK, Chen LC, Choudhury M, Ganju S, Mahajan V, Sinha A, et al. Financing health care for all: challenges and opportunities. Lancet. 2011;377:668-79

6. Million Death Study Collaborators. Changes in causespecific neonatal and 1-59-month child mortality in India from 2000 to 2015: a nationally representative survey. Lancet. 2017;390:1972-80. 